\title{
Éducation géographique et Révolution nationale. La géographie scolaire au temps de Vichy
}

Geographic Education and the National Revolution. School Geography under the Vichy Regime

Geografieausbildung und nationale Revolution. Das Schulfach Erdkunde unter der Vichy-Regierung

Educación geográfica y Revolución nacional : la geografía escolar en tiempos de Vichy

\section{Jean-Pierre Chevalier}

\section{OpenEdition}

Journals

Édition électronique

URL : https://journals.openedition.org/histoire-education/1356

DOI : $10.4000 /$ histoire-education. 1356

ISSN : 2102-5452

Éditeur

ENS Éditions

\section{Édition imprimée}

Date de publication : 1 janvier 2007

Pagination : 69-101

ISBN : 978-2-7342-1076-4

ISSN : 0221-6280

Référence électronique

Jean-Pierre Chevalier, «Éducation géographique et Révolution nationale. La géographie scolaire au temps de Vichy », Histoire de l'éducation [En ligne], 113| 2007, mis en ligne le 01 janvier 2012, consulté le 20 mai 2021. URL : http://journals.openedition.org/histoire-education/1356 ; DOI : https://doi.org/ 10.4000/histoire-education.1356 


\section{ÉDUCATION GÉOGRAPHIQUE ET RÉVOLUTION NATIONALE La géographie scolaire au temps de Vichy}

par Jean-Pierre CHEVALIER

Les grands événements militaires semblent marquer l'histoire de l'enseignement de la géographie en France. En 1870, la défaite apporte un argument supplémentaire à ceux qui militaient en faveur de son enseignement depuis quelques années. Le rapport de Himly et Levasseur de 1871 fait alors date, davantage que les écrits de Levasseur antérieurs à la guerre. Pour la période qui suit, l'historiographie présente communément la préparation de la Revanche comme une des finalités de l'enseignement géographique et la guerre de 1914-1918 comme le fruit d'une éducation patriotique largement portée par la géographie scolaire. En revanche, pour les historiens de la géographie, la Seconde Guerre mondiale, «l'étrange défaite » et la France de Vichy apparaissent souvent comme un simple intermède, une coupure moins importante pour l'épistémologie de la géographie que l'arrivée de la New Geography dans les années 1960, une parenthèse sans conséquence durable sur la géographie scolaire. Pourtant, les programmes de géographie du primaire et du secondaire sont alors réécrits, la licence et l'agrégation de géographie sont mises en place, pendant que l'État français met l'université sous contrôle et que les géographes poursuivent leurs recherches, collaborent, résistent (1) ou se taisent.

Le faible intérêt pour l'histoire de la géographie durant ces années peut s'expliquer par le fait que l'évolution des thèmes de recherche des géographes s'inscrit dans un processus long, qui enjambe les

(1) Jean Gottmann, «French geography in war time », Geographical Review, $\mathrm{n}^{\circ}$ 36, 1946, pp. 80-91; Aimé Perpillou, « Geography and geographical studies in France during the war and the occupation », The Geographical Journal, 1, vol. CVII, 1946, pp. 50-59. 
années de guerre. Les bouleversements des programmes, en particulier dans l'enseignement secondaire, pourraient apparaître comme un simple épiphénomène si l'on acceptait l'hypothèse que ces transformations seraient sans racines antérieures, un épisode que la France républicaine effacerait bien vite à la Libération. À l'inverse, on peut considérer les contenus d'enseignement mis en avant au temps de la Révolution nationale comme une sorte de « test acide » révélateur des vertus prêtées à l'enseignement de la géographie. Test paradoxalement réussi, puisque ce dernier sort renforcé de ces années noires. Ceci doit se comprendre dans un système mettant en relation l'institution scolaire, la géographie appliquée, la géographie universitaire et la volonté des gouvernants.

\section{LA LENTE ÉVOLUTION DES THÉMATIQUES DE RECHERCHE}

Peu nombreux sont ceux qui, comme Marie-Claire Robic (1), Jean-Louis Tissier (2) ou Olivier Dumoulin (3), se sont intéressés aux géographes durant ces durs moments. En effet, les années 1939-1945 sont souvent enjambées par les historiens de la géographie française. Marie-Claude Blanc-Chaléard compare dans le détail les contenus des manuels scolaires de géographie d'avant-guerre et d'aprèsguerre, mais n'a pas de regard particulier sur ceux qui ont été publiés sous Vichy (4). Paul Claval, dans son Histoire de la géographie fran-

(1) Marie-Claire Robic, «Le fardeau du professeur », EspacesTemps, n 68-6970, 1998, pp. 158-170.

(2) Jean-Louis Tissier, « Rendez-vous à Uriage (1940-1942). La fonction du terrain au temps de la Révolution nationale ", in Guy Baudelle, Marie-Vic OzoufMarignier, Marie-Claire Robic (dir.), Géographes en pratiques (1870-1945). Le terrain, le livre, la cité, Rennes, Presses universitaires de Rennes, 2001, pp. 342-351; Cyril Gosme, Jean-Louis Tissier, « Le Territoire de la France, laboratoire de la géographie française », in Marie-Claire Robic (dir.), Couvrir le monde Un grand XXe siècle de géographie française, Paris, ADPF, ministère des Affaires étrangères, 2006, pp. 127-145.

(3) Olivier Dumoulin, « À l'aune de Vichy? La naissance de l'agrégation de géographie », in André Gueslin (dir.), Les Facs sous Vichy, Étudiants, universitaires et universités de France pendant la Seconde Guerre mondiale. Actes du colloque des universités de Clermont-Ferrand et Strasbourg (novembre 1993), Clermont-Ferrand, Institut d'études du Massif Central/Université Blaise Pascal, collection " Prestige ", fasc. 6, 1994, pp. 23-38.

(4) Marie-Claude Blanc-Chaléard, La vision du monde extérieur dans les manuels de géographie avant et après la Seconde Guerre mondiale, DEA, Fondation nationale 
çaise de 1870 à nos jours (1), fait un peu de même, si l'on s'en tient aux titres de ses chapitres: après avoir présenté la géographie de l'entre-deux-guerres dans ses chapitres 6 et 7, il passe à celle du « second après-guerre » dans les chapitres 8 et 9 . Il note au passage que « [1]a préparation des thèses mobilise l'énergie des jeunes collègues durant de longues années, huit ou dix souvent. Les sujets restent toujours dans le cadre régional, mais leur aspect évolue. Dans les années 1940, certains traitent à la fois des aspects physiques et humains de l'aire retenue: Max Derruau en fournit le meilleur exemple » (2). En effet, au regard des publications, et en particulier des thèses, il semble que rien ne change du côté de la géographie universitaire, ou plus exactement que les années de guerre sont à situer dans un long processus d'évolution des thématiques de recherche en géographie (3).

À partir du dépouillement des titres de mémoires de diplômes d'études supérieures publiés par les Annales de géographie, André Meynier constate après-guerre un phénomène à inscrire dans le temps long: le déclin des recherches en géographie physique (tableau 1) (4).

Tableau 1. Les thématiques de recherche dans les années 1938-1951

\begin{tabular}{|l|c|c|c|c|}
\hline \multicolumn{1}{|c|}{ Années de publication } & $1938-1941$ & $1942-1945$ & $1946-1949$ & $1950-1951$ \\
\hline $\begin{array}{l}\text { Mémoires de géographie } \\
\text { régionale }\end{array}$ & $44 \%$ & $42 \%$ & $31 \%$ & $22 \%$ \\
\hline $\begin{array}{l}\text { Mémoires de géographie } \\
\text { physique }\end{array}$ & $2 \%$ & $10 \%$ & $13 \%$ & $7 \%$ \\
\hline $\begin{array}{l}\text { Mémoires de géographie } \\
\text { humaine }\end{array}$ & $54 \%$ & $48 \%$ & $56 \%$ & $71 \%$ \\
\hline
\end{tabular}

(Source: Données élaborées par A. Meynier)

des sciences politiques, 1986, 176 p.; Id., « La vision du monde dans les manuels de géographie français autour de la Seconde Guerre mondiale », L'Espace géographique, $\mathrm{n}^{\circ}$ 4, 1988, pp. 289-298.

(1) Paul Claval, Histoire de la géographie française de 1870 à nos jours, Paris, Nathan, coll. «Université », 1998, 544 p.

(2) Ibid., p. 241.

(3) J. Gottmann, op. cit.

(4) André Meynier, «L'unité de la géographie en péril? », Bulletin de la Société des professeurs d'histoire et de géographie de l'enseignement public, $\mathrm{n}^{\circ} 136$, novembre 1953, pp. 91-93. 
Si l'on se situe entre les bornes 1938 et 1951, on assiste à un lent déclin des travaux classés par A. Meynier en géographie régionale et à un progrès symétrique des mémoires soutenus en géographie humaine pure. Cependant, les années 1942-1945 se situent plutôt à contre-courant, avec une progression nette des recherches en géographie physique pure et un recul passager des mémoires soutenus en géographie humaine. Les fluctuations ne sont pas considérables, mais elles peuvent avoir du sens, surtout si on compare la répartition des thèmes de recherche de 1942-1945 à celle des mémoires soutenus par les étudiants entrés à l'université après-guerre, en 1950-1951.

Cette évolution des thématiques de recherche ne peut s'expliquer uniquement par des changements de centre d'intérêt, par une évolution épistémologique de la géographie. Il faut aussi tenir compte des difficultés liées à la guerre: étudiants et enseignants mobilisés, puis souvent prisonniers, comme Henri Enjalbert, restrictions de circulation, censure, paupérisation générale. Tout cela se conjugue avec les impossibilités que l'on imagine pour les enquêtes de terrain, ainsi qu'avec la raréfaction du papier qui entraîne progressivement un déclin des publications (1) et des thèses (tableau 2). Aimé Perpillou et Jean Gottmann rappellent que Théodore Lefebvre, professeur à Poitiers, a été emprisonné puis tué par les nazis, que Jacques Ancel, professeur à la Sorbonne, est mort à Drancy, que René Musset, professeur à Caen, a été enfermé deux ans à Buchenwald, qu'à Clermont-Ferrand Henri Baulig a été emprisonné plusieurs mois par la Gestapo (2).

(1) L'Information géographique signale six titres en 1939: Henri Collin Delavaud, Vers l'inconnu. Les hommes à la découverte de la terre; Henri Lhote, Le Sahara, désert mystérieux; Jean-Albert Vellard, Une civilisation du miel (Les Indiens Guayakis du Paraguay); Marcel Blanchard, Géographie des chemins de fer; René Clozier, Les étapes de la géographie; Marcel-Adolphe Hérubel, Les ports maritimes. Un seul titre est signalé en 1941 : Albert Demangeon, Jacques Milley, Éléments de géographie par l'image. Quatre ouvrages sont indiqués en 1942: Pierre George, À la découverte du pays de France. La nature et les travaux des hommes; Jean-François Gravier, Régions et nations; Yann-Marie Goblet, La formation des régions. Introduction à la géographie économique de la France; André Cholley, Le guide de l'étudiant en géographie. Deux en 1943: Maximilien Sorre, Les fondements biologiques de la géographie humaine. Essai d'une écologie de l'homme; André Siegfried, Vue générale de la Méditerranée. Aucun en 1944.

(2) J. Gottmann, op. cit.; A. Perpillou, op. cit. 
Tableau 2. Les thèses soutenues et leur publication, 1939-1945 (1)

\begin{tabular}{|c|l|}
\hline Années & \multicolumn{1}{|c|}{ Thèses publiées } \\
\hline 1939 & $\begin{array}{l}\text { Lucien Gachon, Les Limagnes du Sud et leurs bordures monta- } \\
\text { gneuses, étude de géographie physique et humaine. } \\
\text { Jean Robert, La maison rurale permanente dans les Alpes fran- } \\
\text { çaises du Nord. } \\
\text { René Clozier, La gare du Nord, étude de géographie urbaine. Les } \\
\text { causses du Quercy, contribution à la géographie physique d'une } \\
\text { région calcaire (thèse secondaire). } \\
\text { Aimé Perpillou, Le Limousin, étude de géographie physique. } \\
\text { Jacques Weulersse, Le Pays des Alaouites. } \\
\text { Francis Ruellan, Le Kwansaï, étude géomorphologique d'une } \\
\text { région japonaise. } \\
\text { Lucien Goron, Les Pré-Pyrénées ariégeoises et garonnaises. } \\
\text { Essai d'étude géomorphologique d'une région montagneuse. } \\
\text { Éric Dardel, La pếche harenguière en France. } \\
\text { Louis Papy, La Côte atlantique de la Loire à la Gironde. Tome 1: } \\
\text { Les aspects naturels; Tome 2: L'Homme et la mer. } \\
\text { Jean Dresch, Recherches sur l'évolution du relief dans le Massif } \\
\text { Central du Grand Atlas, le Haouz et le Sous. } \\
\text { Maurice Le Lannou, Pâtres et paysans de la Sardaigne. } \\
\text { Antoine Albitreccia, La Corse, son évolution au XIXe et au début } \\
\text { du XXe siècle. } \\
\text { Georges Baeckeroot, Oesling et Gutland. Morphologie du bassin } \\
\text { ardennais et luxembourgeois de la Moselle. } \\
\text { Yves Urvoy, Les bassins du Niger. Étude de géographie physique } \\
\text { et de paléogéographie. } \\
\text { Aucune } \\
\text { Paul Veyret, Les Pays de la Moyenne Durance alpestre (Bas- } \\
\text { Embrunais, Pays de Seyne, Gapençais, Bas-Bochaine). Étude } \\
\text { géographique. } \\
\text { Aucune }\end{array}$ \\
\hline
\end{tabular}

De 1942 à 1945 les soutenances de thèse se font rares: on imagine assez aisément que René Clozier aurait eu beaucoup de mal à conduire ses enquêtes de géographie humaine sur la gare du Nord au temps de l'occupation ou que le révérend Baeckeroot était dans l'impossibilité de poursuivre ses travaux de géomorphologie au Luxembourg. La guerre interdit de nombreux travaux de recherche.

(1) D'après leur relevé dans L'Information géographique entre 1939 et 1940 et d'après leur recension in Couvrir le monde..., op. cit. 
Les thèses soutenues au lendemain de la guerre correspondent donc à des espaces restés accessibles et à des recherches dont les thématiques sont fortement marquées par la géographie physique (1).

La situation est plus différenciée du côté des revues scientifiques de géographie. Certaines revues cessent de paraître plus ou moins rapidement, d'autres poursuivent leur publication tout au long de la guerre. De 1940 à 1946 la Revue de la Société de géographie cesse de paraître et fusionne avec les Annales de géographie. L'Information géographique interrompt brusquement sa publication en avril 1942 et ne reparaît qu'à partir de juin 1945.

Les Annales de géographie sont quelque peu touchées à partir de 1943 par les restrictions; en conséquence, le texte se continue sur les couvertures par suite du manque de papier. Son comité de patronage est de moins en moins nombreux. Composé de douze membres en 1937 selon le $\mathrm{n}^{\circ} 259$, ils n'en comportent plus que six en janvierfévrier 1943. Mais, curieusement, la liste des pays à tarif postal réduit pour acheminer la revue ne change guère. Certes, l'Autriche est supprimée de cette liste, de même que l'Éthiopie. Mais la Tchécoslovaquie devient une curieuse Tchéco-slovaquie et, en 1943, la Pologne et la Yougoslavie sont toujours nommées, alors qu'elles aussi ont cessé d'exister en tant qu'États. Ainsi les revues de géographie, quand elles paraissent, semblent figées, comme si la guerre n'avait pas eu lieu, ou plutôt n'était pas encore conclue.

Les publications de province semblent encore moins touchées par les restrictions. Ainsi, le Bulletin de la Société languedocienne de géographie échappe pratiquement aux restrictions en matière de papier (2). Certes, il est écrit dans la revue tout au long de la guerre, de 1940 jusqu'en 1945, que les prescriptions gouvernementales l'obligent à réduire de $50 \%$ son nombre de pages. Mais fin 1942 et début 1943, le bulletin obtient de gros contingents de papier lui permettant de publier sur plus de 200 pages (tableau 3).

(1) Jean Chardonnet, Le Relief des Alpes du Sud, 1947; Jacqueline BeaujeuGarnier, Le Morvan, étude morphologique, 1948; Jean Tricart, La Partie orientale du Bassin de Paris, étude morphologique, 1949; Max Derruau, La Grande Limagne auvergnate et bourbonnaise, 1949.

(2) Notons deux articles sous la signature de J. Gottmann: dans le tome XI de 1940, un hommage nécrologique à Jules Sion, et, dans le $1^{\text {er }}$ fascicule de 1941, un article rédigé avec Pierre Gourou pour la nécrologie d'A. Demangeon. 
Tableau 3. La pagination du Bulletin de la Société languedocienne de géographie (1940-1945)

\begin{tabular}{|c|l|l|}
\hline Année & Fascicules publiés & Nombre de pages des publications de l'année \\
\hline 1940 & tome XI & 64 pages \\
\hline 1941 & 2 fascicules & $68+68=136$ pages \\
\hline 1942 & 2 fascicules & $68+132=200$ pages \\
\hline 1943 & 2 fascicules & $144+52=196$ pages \\
\hline 1944 & 2 fascicules & $86+86=172$ pages \\
\hline 1945 & 2 fascicules & $64+78=142$ pages \\
\hline
\end{tabular}

Les conditions matérielles des équipes rédactionnelles, leurs orientations face à la censure, les restrictions de papier, plus ou moins grandes suivant les régions, tous ces facteurs contribuent à différencier l'évolution de ces publications. Une étude comparée de l'ensemble des revues de géographie pendant les années de guerre reste à mener pour dégager des tendances plus générales, mais en ces temps de crise, c'est la stabilité de la géographie universitaire qui apparaît comme la trame de fond de ces publications. Les articles se fondent sur des travaux antérieurs à la guerre.

Dans ce contexte, la création de la licence de géographie en 1941 et celle de l'agrégation de géographie en 1943 apparaissent comme le couronnement d'une discipline installée. Pourtant la refonte des programmes de géographie de l'enseignement primaire et secondaire invite à penser que les contenus d'enseignement de cette discipline dépendent des fluctuations politiques et que les promoteurs de la Révolution nationale comptaient bien s'appuyer sur l'enseignement de la géographie pour promouvoir leur idéologie.

\section{LA GÉOGRAPHIE SCOLAIRE REFORMATÉE}

À partir de septembre 1940, le contexte politique offre des opportunités pour des transformations technocratiques. Pour les promoteurs de la géographie, la période qui s'ouvre avec le gouvernement de Vichy ressemble à celle qui a suivi la défaite de 1870 . Le développement de l'enseignement de la géographie est à nouveau présenté comme une nécessité pour redresser le pays. L'accent est mis sur le ruralisme, le passéisme, le naturalisme, mais pointe le goût pour 
l'action volontaire, et l'aménagement du territoire se manifeste aussi. Le régime de Vichy multiplie donc les mesures pour développer et encadrer la diffusion de la géographie. Dès septembre 1940, les programmes de géographie du primaire sont revus (1). En 1941, c'est la création de la licence de géographie (2) et la modification des programmes et des horaires de l'enseignement dans le secondaire (3). En 1942, un nouveau plan d'étude est décidé pour l'enseignement primaire (4). Enfin, en 1943, l'agrégation de géographie est créée, après une première tentative avortée en 1941 (5).

De l'enseignement primaire à l'enseignement secondaire et universitaire, l'ensemble de l'enseignement de la géographie est donc remodelé à l'époque de Vichy. La place de la géographie à l'école est renforcée. C'est le fruit d'une action entreprise depuis longtemps par des géographes, et conduite pendant ces années par deux professeurs à la Sorbonne, Emmanuel de Martonne et André Cholley (6). Mais le contenu des programmes est aussi revu en fonction des options idéologiques de la Révolution nationale.

\section{Attacher les enfants au pays natal}

La mise en place de l'enseignement de la géographie au cours du $\mathrm{XIX}^{\mathrm{e}}$ siècle s'est faite parallèlement à la construction des États nationaux (7). À l'époque de la III République, à côté de ses intérêts pratiques, la géographie est apparue comme une des disciplines scolaires concourant à la construction du sentiment patriotique. C'est une des finalités, au moins implicites, de son enseignement (8). Mais après la

(1) Arrêté du 14 septembre 1940 et circulaire du 9 octobre 1940.

(2) Décret du 28 avril 1941.

(3) Arrêté du 22 septembre 1941 (classes de sixième) sur les exemples locaux.

(4) Instructions du 5 mars 1942.

(5) Arrêté du 28 septembre 1943; les modalités seront précisées par la circulaire du 28 mars 1944.

(6) O. Dumoulin, op. cit.

(7) Horacío Capel, « Institutionalization of Geography and Strategies of Change ", in David Ross Stoddart (ed.), Geography, ideology and social concern, Cambridge, Basil Blackwell, 1981, pp. 37-69.

(8) Jean-François Chanet, L'École républicaine et les petites patries, Paris, Aubier, 1996, 426 p.; Anne-Marie Thiesse, Ils apprenaient la France. L'exaltation des régions dans le discours patriotique, Paris, Éditions de la Maison des sciences de l'homme, 1997, $132 \mathrm{p}$. 
Première Guerre mondiale, les enseignants, en particulier ceux du premier degré, sont devenus très sensibles aux courants pacifistes (1). C'est dans ce contexte que, très rapidement, le maréchal Pétain s'attaque à l'enseignement primaire. Georges Ripert, secrétaire d'État à l'Instruction et à la Jeunesse, ferme les écoles normales primaires, foyers d'esprit républicain, et révoque les instituteurs francs-maçons. Dans le domaine de l'enseignement de la géographie, l'accent est alors mis sur l'étude de la France, ce qui n'est pas une nouveauté, sur la France d'outre-mer, comme est appelé l'empire colonial, et sur l'étude du milieu local, éventuellement à l'occasion de sorties :

« Géographie.

$1^{\circ}$ Principaux traits de la géographie de la France. Rappeler les notions essentielles de relief, du climat, des fleuves, de la population et des aspects économiques tout en consolidant la connaissance de la nomenclature par l'usage constant de la carte;

$2^{\circ}$ La France d'outre-mer;

$3^{\circ}$ Géographie locale. On étudiera la géographie de la petite région; cette étude sera éventuellement l'occasion d'excursions dirigées par les maîtres ou de promenades suggérées aux élèves.

Fait à Vichy, le 14 septembre 1940.

Signé : Georges Ripert. »

Cette orientation est poursuivie et explicitée par Jérôme Carcopino, secrétaire d'État à l'Éducation nationale et à la Jeunesse dans le gouvernement de l'amiral Darlan. Il distingue nettement deux niveaux d'échelle à privilégier: le canton ou le petit pays au cours moyen, la province dans les classes préparatoires au certificat d'études primaires.

«Mais la grande nouveauté du programme du cours moyen réside dans l'étude méthodique du milieu local, et non précisons-le bien, du milieu régional. Il ne s'agit que du petit pays, de la petite cellule géographique, Lodévois, Trièves, Aunis ou campagne de Caen, par

(1) Jean-Pierre Chevalier, Du côté de la géographie scolaire. Matériaux pour une épistémologie et une histoire de l'enseignement de la géographie à l'école primaire en France, rapport de synthèse en vue d'une HDR, Université Paris 1, 2003, pp. 307-311; Olivier Loubès, «L'étrange défaite de la patrie à l'école primaire en France entre 1918 et $1940 »$, Historiens \& géographes, ${ }^{\circ} 390$, avril 2005, pp. 193-202.

(2) Arrêté du 14 septembre 1940 sur l'enseignement primaire. 
exemple, qui peut embrasser un ou plusieurs cantons. Cette étude pourrait s'étaler sur les deux derniers mois de l'année scolaire, plus favorables aux promenades scolaires qui en fourniront la substance et non le complément; elle doit partir de la commune elle-même, certes connue, mais mal observée, et insister beaucoup sur ses divers aspects. L'on trouvera dans l'observation attentive de l'horizon familier une mine inépuisable de faits concrets et précis se rapportant aux traits physiques (site, sol, climat, rivière, calendrier agricole) comme aux caractères humains (éléments, variation de la population, son mode de groupement et de dispersion, étude de la maison) et enfin aux activités économiques (observation du cadastre, du domaine paternel, étude des cultures et des productions, foires, industries et commerce, visite de la gare, etc.). Si l'enfant est un citadin, l'étude de la ville, en commençant par le quartier, peut facilement mener à l'analyse du site, du développement et du plan de la cité, de sa population, de son rôle administratif, commercial, industriel. Et l'on ne saurait trop encourager instituteurs et institutrices à établir, et il en est déjà de bien faites, une monographie de leur commune et de leur canton.

En attendant, d'autre part, que s'éditent des cahiers géographiques destinés à cette observation du milieu local - et il en est déjà de parus - il sera bon de demander à l'élève de tenter, lui aussi, sur un cahier spécial, une petite monographie de sa commune avec questionnaires, croquis, dessins, graphiques, observations sur ce qu'il aura appris en classe et vu en classe-promenade » (1).

Les monographies communales ne sont pas une nouveauté. C'est un genre administratif auquel les instituteurs ont été souvent appelés à contribuer, en particulier à l'occasion des expositions internationales de 1889 et 1900 . C'est aussi une activité pédagogique dans la filiation des leçons de choses. Mais pour les gouvernants de Vichy, la promotion du local et l'accent sur les monographies ne sont pas un simple choix pédagogique. Il s'agit de décliner, via la géographie, la Révolution nationale. L'accent sur les racines ancestrales et terriennes des petits pays doit concurrencer l'échelon républicain du département, et aussi la région naturelle, de dimension plus vaste, trop abstraite pour de jeunes enfants, pas assez enracinée pour le nouveau régime.

(1) Instructions du 5 mars 1942 relatives au nouveau plan d'études des écoles primaires élémentaires, signées par Jérôme Carcopino, partie V: Géographie. 
Dans le second cycle primaire, c'est-à-dire dans les deux années conduisant à l'examen du certificat d'études l'enseignement de la géographie est également recadré. Une année est consacrée à la géographie du monde, une autre à celle de la France (1). Le développement des instructions officielles le plus étoffé est, ici aussi, consacré à la région où vivent les élèves, qui « servira alors de couronnement à l'étude des régions naturelles $»$ : derrière ces mots, c'est en fait le cadre de l'ancienne province qui est privilégié.

"Le mot région n'a pas du reste ici le même sens que dans l'expression de "région naturelle". Il s'agit de la région fondée autant sur l'histoire que sur la géographie. Il ne peut être question, en effet, de demander à un Champenois ou à un Auvergnat d'étudier tout le bassin parisien ou tout le Massif Central, mais seulement la Champagne ou l'Auvergne.

Après avoir de façon précise situé le milieu provincial dans le cadre plus vaste des grandes divisions naturelles, la région ainsi comprise, et qui peut couvrir un ou plusieurs départements, sera étudiée non seulement dans ses cadres géographiques usuels (traits physiques, vie humaine, activité économique), mais aussi dans les aspects proprement dits de la vie régionale (mœurs, coutumes, traditions). L'on ne manquera pas de s'intéresser au folklore local, de chercher le souvenir des vieilles chansons, des vieux usages, des vieilles légendes. L'attrait de ces recherches sur l'originalité du terroir ne peut manquer de contribuer à retenir les enfants dans leur petite patrie, tout en alimentant leur curiosité intellectuelle et même artistique. Mais l'on observera que la vie régionale n'est qu'un des multiples aspects de notre existence nationale, de son unité cimentée dans la diversité provinciale, par tant de siècles de vie commune par tant d'affinités naturelles qui rendent nos provinces complémentaires et solidaires » (2).

On peut parler ici de dialectique du local et du national, ou d'une tentative de combinaison des racines terriennes traditionnelles et de l'éducation patriotique, comme l'ont souligné Anne-Marie Thiesse et

(1) Depuis 1941 les écoles primaires supérieures ont été intégrées à l'enseignement secondaire sous la forme de collèges.

(2) Instructions du 5 mars $1942 \ldots$, loc. cit. 
Jean-François Chanet (1). Par le biais de l'étude du local et de l'Empire, la nation est alors traitée au niveau de l'idéal, la question du territoire français en Europe étant ainsi quelque peu mise entre parenthèses. Avec ces approches monographiques du petit pays ou de la province historique, les programmes de Vichy gardent en arrière-fond implicite la référence à la pédagogie intuitive et à la démarche scientifique inductive, comme les programmes de 1923 (2) et les prescriptions antérieures. Mais ils s'en distinguent par la trame idéologique de la Révolution nationale: le retour à la terre, l'enracinement dans la France traditionnelle. L'idée de rupture est expressément développée dans la première partie de ces instructions. Carcopino fait référence à la réduction structurelle de l'enseignement primaire, privé de ses écoles primaires supérieures et de ses écoles normales, qui vise désormais principalement le certificat de fin d'études. Les valeurs promues par les gouvernants de Vichy éclairent ces programmes:

« Pour répondre non seulement aux besoins de chacun, mais aussi aux besoins de la nation, une réforme de l'enseignement ne peut manquer d'accentuer le caractère pratique de la formation donnée aux adolescents. Le nouveau plan d'études désigne avec précision les conditions particulières, locales et régionales, auxquelles nos élèves doivent adapter leur activité, et il aura pour effet d'attacher davantage les enfants au pays natal, de prévenir les déplacements dont la campagne et toute la vie française ont jusqu'ici tellement souffert » (3).

La partie sur la géographie dans le second cycle du primaire, qui conduit au certificat de fin d'études, peut alors se conclure par cette envolée reprenant l'expression d'Octave Gréard sur ce qu'il n'est pas permis d'ignorer (4), qu'elle relativise néanmoins par rapport aux finalités patriotiques de l'enseignement:

(1) J.-F. Chanet, op. cit.; A.-M. Thiesse, op. cit.

(2) L'ensemble des programmes de géographie du primaire jusqu'en 1990 sont reproduits dans le volume annexe de la thèse de Monique Benoît, L'enseignement de la géographie à l'école primaire, 1867-1991, Université Paris 1, 1992, 3 vol., 203 p., 239 p., 258 p.

(3) Instructions du 5 mars 1942, signées par J. Carcopino, partie I: Principes généraux et organisation des études.

(4) Instruction générale adressée à MM. les Inspecteurs de l'instruction primaire [du département de la Seine] sur la mise à exécution du Règlement d'organisation pédagogique des Écoles, 17 août 1868, signée par Octave Gréard. 
« Ainsi en quittant l'école primaire, le jeune Français connaîtra de tout le vaste monde, ce qu'il n'est pas permis d'ignorer; mais mieux encore, il aura appris à connaître sa patrie. L'enseignement de la géographie aura finalement rejoint la constante leçon de patriotisme que lui auront donnée ses études d'histoire, de morale et de langue française » (1).

Jérôme Carcopino et ses collaborateurs conçoivent clairement la géographie scolaire comme une discipline idéologique.

\section{Une augmentation massive de l'horaire de géographie dans le secondaire}

La logique des changements des programmes du secondaire est à première vue fort semblable et, comme dans le primaire, la modification se fait en deux temps. Un premier arrêté de Jérôme Carcopino réoriente les programmes en 1941 (2), puis, en 1943, le ministre Abel Bonnard restructure profondément la cohérence du curriculum de la géographie dans l'enseignement secondaire. Ces programmes ont été étudiés d'abord par Maria-Manuela Costa-Ferreira, ensuite, plus précisément, par Isabelle Lefort (3). En 1943, l'augmentation de l'horaire consacré à la géographie dans l'enseignement secondaire est spectaculaire (tableau 4).

Dans l'enseignement secondaire, l'horaire consacré à l'histoire était traditionnellement plus important que celui attribué à la géographie. En 1941, dans un contexte où l'équilibre général des disciplines est bouleversé (au bénéfice principalement de l'éducation physique), l'horaire de géographie est maintenu, alors que celui de l'histoire est réduit; en 1943, il est considérablement augmenté. Tandis que les programmes de 1938 attribuaient à la géographie un nombre d'heures moitié moindre de celui de l'histoire, ceux de 1943 lui accordent une part égale. « Les nouveaux programmes, par l'augmentation massive

(1) Instructions du 5 mars 1942, partie V: Géographie.

(2) Arrêté du 22 septembre sur les programmes de l'enseignement secondaire.

(3) Les programmes de géographie du secondaire de 1874 à 1963 sont reproduits dans le tome annexe de la thèse de doctorat d'Isabelle Lefort, Géographie savante Géographie scolaire (1870-1970). Éléments pour une histoire de la pensée géographique, Université Paris 1, 1990, 2 vol. 
Tableau 4. Les horaires hebdomadaires d'enseignement de la géographie et de l'histoire dans l'enseignement secondaire (programmes de 1938, 1941 et 1943)

\begin{tabular}{|l|c|c|c|}
\hline Classe & $\begin{array}{c}\text { Horaire de 1938 } \\
\text { Géographie } \\
\text { + Histoire }\end{array}$ & $\begin{array}{c}\text { Horaire de 1941 } \\
\text { Géographie } \\
\text { + Histoire }\end{array}$ & $\begin{array}{c}\text { Horaire de 1943 } \\
\text { Géographie } \\
\text { + Histoire }\end{array}$ \\
\hline $6^{\mathrm{e}}$ & $1+1,5$ & $1+1$ & $1+1$ \\
\hline $5^{\mathrm{e}}$ & $1+1,5$ & $1+1$ & $1,5+1,5$ \\
\hline $4^{\mathrm{e}}$ & $1+2$ & $1+1$ & $2+2$ \\
\hline $3^{\mathrm{e}}$ & $1+2$ & $1+1,5$ & $2+2$ \\
\hline $2^{\text {de }}$ & $1+2$ & $1+2$ & $2+2$ \\
\hline $1^{\text {re }}$ & $1+2,5$ & $1+2$ & $2+2$ \\
\hline $\begin{array}{l}\text { Philosophie et } \\
\text { Mathématiques }\end{array}$ & $7+13,5$ & $7+2$ & $2+2$ \\
\hline Total & & 71,5 & $12,5+12,5$ \\
\hline
\end{tabular}

qu'ils accordent à l'horaire de la géographie, consacrent la valeur éducative de cette discipline et reconnaissent son efficacité pour la formation spirituelle des temps modernes », est-il écrit dans l'annexe à l'arrêté du 5 août 1943 (1).

Les changements ne sont pas que quantitatifs, l'ordre du curriculum de géographie étant aussi bouleversé. Désormais, la géographie générale couronne les deux cycles d'études de géographie au lieu d'en être l'amorce. L'accent est mis sur le concret, qui prime sur les généralisations, et l'ensemble de ces études est fortement centré sur la France et son empire colonial (tableau 5).

Isabelle Lefort souligne que le curriculum de 1943 (allant des « leçons de choses » aux géographies particulières, puis à la géographie générale) relève d'une organisation inductive des programmes. Sur ce point, il diffère fortement des précédents, qui relevaient d'une logique plutôt déductive (de la géographie générale aux géographies particulières), depuis 1902 notamment. Ce constat est à mettre en relation avec l'accent porté sur le caractère « concret » des objets

(1) L'information universitaire, $\mathrm{n}^{\circ} 1157,11-25$ septembre 1943, cité par M.-M. Costa M. D. A. Ferreira, Les programmes de géographie dans l'enseignement secondaire en France (début du XIXe siècle - 1980), doctorat de $3^{\mathrm{e}}$ cycle, Université Paris 7 , 1980. 
d'étude de la géographie: le mot revient comme un leitmotiv. En classe de sixième, «l'enseignement de la géographie doit, avant tout, garder un caractère concret» (programme de 1941). "L'enseignement de la géographie se doit d'être évocateur, vivant et concret [...]. Afin de rester concret, de s'étayer solidement sur le réel, le professeur appuiera son enseignement sur des cartes, des croquis au tableau, des gravures... » (Instructions générales de 1943). L'idée que la géographie est une discipline du concret est probablement une des raisons qui ont incité les responsables de Vichy à renforcer son enseignement, ce qui n'empêche pas les géographes d'affirmer le caractère scientifique de leur discipline. La géographie générale tient une place importante et repose, si ce n'est toujours sur des règles, du moins sur des schémas explicatifs globaux. L'induction a comme horizon la généralisation.

Ces réformes renforcent aussi le caractère franco-centré des programmes. Dans le curriculum de 1938, deux années étaient consacrées à la géographie de la France, dans celui de 1943, la France est vue en sixième, cinquième, quatrième et première. Elle est également présente les autres années au travers de ses possessions outre-mer.

« Voilà pourquoi, en sixième et en cinquième, la France servira de point de départ afin de rester le terme de comparaison. La France au sens large, bien entendu, flanquée de ses prolongements coloniaux où vivent certains de nos élèves, où d'autres seront un jour appelés. Il est indispensable que, dès les débuts, nos enfants soient dressés à penser sur le plan mondial qui est le nôtre. Dans les classes suivantes, la Terre française et les Terres impériales ne disparaîtront jamais de l'horizon de nos élèves: elles demeureront toujours présentes comme termes de rappel et de comparaison (Instructions générales de 1943)».

Dès 1940 , c'est-à-dire dès le début de la mise en œuvre du programme de 1938, René Clozier s'était fait, au nom de l'Association de géographes français, le porte-parole d'une demande de refonte des programmes du second cycle du secondaire, en particulier du programme de seconde, "figé dans l'archaïsme des temps prégéographiques »(1). Un questionnaire d'enquête avait été diffusé

(1) Séance du 9 novembre 1940 de l'Association de géographes français, Bulletin de l'Association de géographes français, $\mathrm{n}^{\circ}$ 132-133, pp. 72-74. 


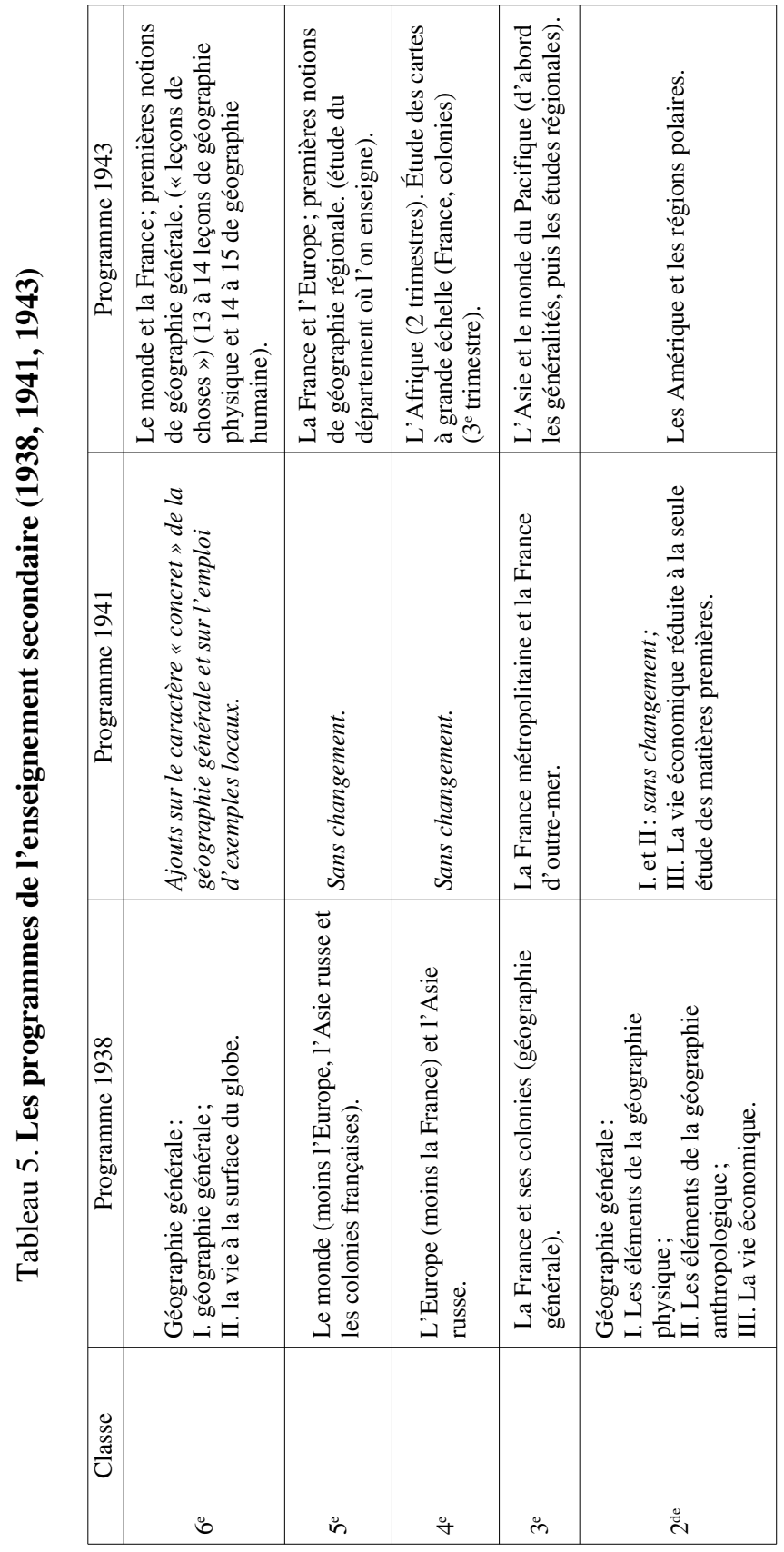




\begin{tabular}{|c|c|c|}
\hline 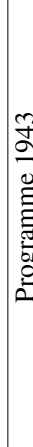 & 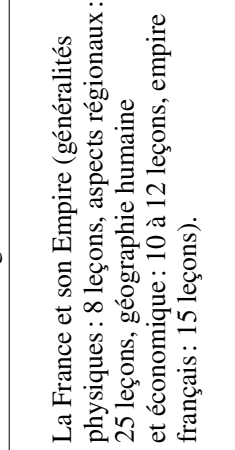 & 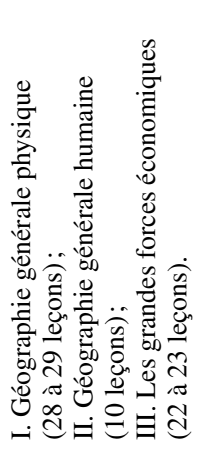 \\
\hline 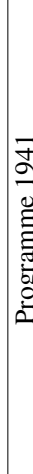 & 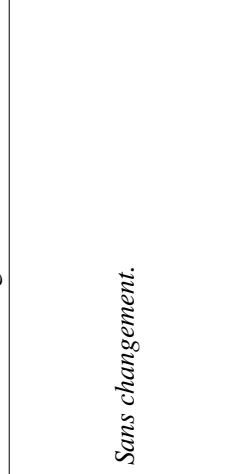 & 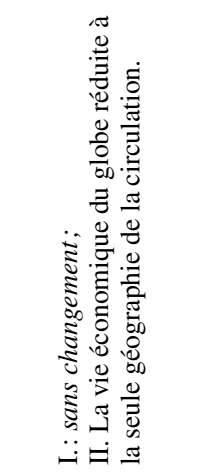 \\
\hline के & 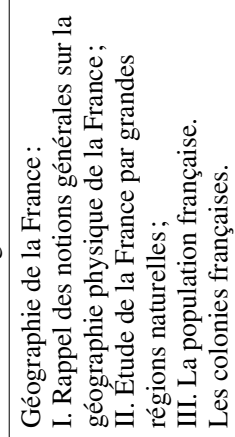 & 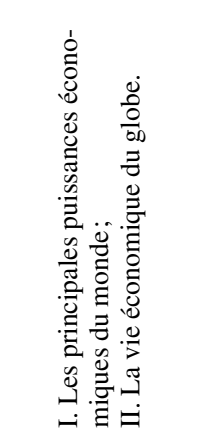 \\
\hline 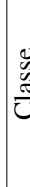 & $\mathscr{\varrho}$ & 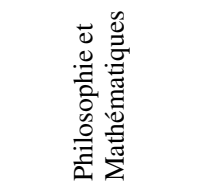 \\
\hline
\end{tabular}


en février 1940 auprès des membres de l'Association (1). Les résultats de ce "référendum » furent communiqués au lendemain de la défaite (2). Les collègues consultés par l'AGF en 1940 réclamaient unanimement trois modifications, qui seront satisfaites par la rédaction du programme de 1943: 1. la suppression ou la réduction de la géographie économique en seconde; 2 . la réduction du nombre de grandes puissances, avec report de la géographie économique en philosophie; 3. une nouvelle rédaction du programme de géographie générale. Certains demandaient aussi le report de la géographie régionale en seconde, la suppression de l'étude régionale pour les empires coloniaux étrangers, d'autres souhaitaient un système de sujets à option en classe terminale. Lors de la discussion, les intervenants se situent plus ou moins implicitement par rapport aux premières mesures du gouvernement du maréchal Pétain. Pierre George fait preuve d'intérêt pour la formation des futurs instituteurs dans les lycées, puisque les écoles normales ont été supprimées. E. de Martonne se demande si la géographie ne pourrait pas intervenir aussi dans les exercices physiques lors des excursions et des camps de vacances, dans le cadre de l'augmentation de l'horaire d'éducation civique voulue par le nouveau régime. L'inspecteur général Henri Boucau s'interroge aussi sur la façon de combiner l'augmentation de l'horaire des exercices physiques (onze heures hebdomadaires) et celui de l'horaire de la géographie: il propose une demi-journée de marche combinée avec une exploration de paysage sur le terrain. Bien qu'il rappelle que « les historiens, qui forment la masse principale des professeurs d'histoire et géographie, ne sont pas favorables à une augmentation du temps consacré à la géographie », il conclut: " La géographie doit jouer son rôle dans la formation des jeunes hommes ».

La place importante prise par la géographie dans les programmes de l'enseignement secondaire en 1943 semble donc résulter de la conjonction opportune du lobby des géographes universitaires de la Sorbonne avec les orientations idéologiques de Vichy. Il en va de même à l'université.

(1) Emmanuel de Margerie, Bulletin de l'Association de géographes français, $\mathrm{n}^{\circ} 126-127$, séance du $1^{\text {er }}$ février 1940, pp. 17-18

(2) René Clozier, «Referendum sur les programmes de Géographie ( $2^{\mathrm{e}}$ cycle) dans l'enseignement secondaire », Bulletin de l'Association de géographes français, $\mathrm{n}^{\circ} 132-133,1940$, pp. $72-74$. 


\section{Création de la licence de géographie en 1941 et de l'agrégation en 1943}

Comme après 1871 , l'importance accordée à la géographie scolaire vient renforcer la place de la géographie à l'université. Pourtant, les relations entre l'université et l'enseignement secondaire ne sont pas toujours simples. Ainsi, dans l'introduction à son Guide de l'étudiant en géographie, A. Cholley, professeur à la Sorbonne, déclare en 1942 souhaiter une réforme qui « libérerait l'enseignement supérieur » de la « lourde hypothèque » que fait porter sur lui la formation des enseignants du secondaire:

« Le but de l'enseignement supérieur doit être avant tout la formation technique des travailleurs qui se consacrent à la recherche scientifique, et leur groupement en vue d'assurer la coordination de leurs efforts.

Dans nos facultés de lettres, en particulier, cette fonction essentielle a été entravée et même faussée par une préoccupation d'ordre pratique : assurer à l'enseignement secondaire, notamment, le recrutement de ses cadres. [...] Nos efforts sont maintenus obligatoirement, ou peu s'en faut, dans les voies traditionnelles imposées par l'enseignement secondaire; les chemins nouveaux, ceux qui conduisent à la découverte, nous sont, sinon à peu près interdits, du moins rendus très difficiles à suivre » (1).

Cholley parle de libérer les universitaires du fardeau de l'enseignement secondaire, mais on peut y voir aussi une habileté pour s'affranchir du partenariat avec les historiens. Décrivant l'histoire de cette période, Olivier Dumoulin parle d'un « combat libérateur » des géographes durant les années 1940-1944 (2). De tels arguments appuient la revendication d'une licence spécifique de géographie, qui facilitera ensuite la création d'une agrégation de géographie; celle-ci, en retour, favorisera le développement autonome de la géographie universitaire.

(1) A. Cholley, Guide de l'étudiant en géographie, Paris, Presses universitaires de France, $1^{\text {re }}$ éd. 1942, p. 1.

(2) O. Dumoulin, op. cit., p. 28. 
Jusqu'en 1940, les facultés de lettres proposaient une licence d'histoire (mention enseignement) comprenant trois certificats d'histoire et un certificat de géographie, et une licence d'histoire et géographie composée de deux certificats d'histoire (moderne et du Moyen-Âge) et de deux certificats de géographie (générale et régionale). Par un décret daté du 28 avril 1941, les géographes obtiennent la création d'une licence de géographie, exact symétrique de la licence d'histoire (mention enseignement), puisque composée de trois certificats de géographie (générale, régionale et cartographie) et d'un certificat d'histoire (1). En 1943, Cholley obtient qu'il n'y ait pas d'épreuve de latin dans la licence de géographie. C'est la seule discipline « littéraire » dans ce cas; les études de géographie s'ouvrent alors plus largement aux anciens normaliens du primaire.

La création de l'agrégation de géographie est issue d'un long combat. Elle a été réclamée par Ludovic Dupeyron à la fin du $\mathrm{XIX}^{\mathrm{e}}$ siècle et revendiquée par certains lors des conférences de 1905. Dans les années 1940, E. de Martonne en est le promoteur principal. Georges Chabot témoigne: "Il y consacra ses forces, son autorité pendant plusieurs années. On serait émerveillé si l'on pouvait rassembler toute sa correspondance à ce sujet $[\ldots]$; il profitait de ses voyages pour contacter, interroger, convaincre les collègues; vis-àvis des ministres, de leurs directeurs, il avait l'autorité que lui conféraient une réputation scientifique mondiale, la présidence de tous les groupements géographiques français et celle de l'Union géographique internationale » (2). Un premier arrêté du 28 avril 1941 instituant une agrégation de géographie, concomitant avec la nouvelle licence, est suspendu par un décret du 16 juin 1942, suite aux interventions de nombreux opposants historiens ou géographes. Raoul Blanchard, Louis Papy, A. Meynier y sont hostiles ou réticents, alors que Pierre Birot et Cholley y sont favorables (3). E. de Martonne multiplie les interventions et les réunions et obtient finalement du ministre Abel Bonnard la publication, le 28 septembre 1943, de

(1) Décret du 28 avril 1941, modifié le 13 août 1945, corrigé le 17 octobre 1945; Georges Chabot, "La nouvelle licence de géographie », L'Information géographique, 1946, n 1, pp. 25-27; J. Beaujeu-Garnier, «Licence d'histoire et... licence de géographie », L'Information géographique, 1949, n 2, pp. 64-66.

(2) G. Chabot, « La genèse de l'agrégation de géographie », Annales de géographie, vol. 85, 1976, pp. 333-340.

(3) Claude Bataillon, «Table ronde imaginaire sur la géographie universitaire française, 1930-1940», Hérodote, $\mathrm{n}^{\circ} 20,1981$, pp. 116-153. 
l'arrêté déclarant qu' «il est institué une agrégation d'histoire et une agrégation de géographie ». Cette séparation entre l'histoire et la géographie n'est pas totale, la géographie étant maintenue dans l'agrégation d'histoire et l'histoire dans l'agrégation de géographie. De plus, comme le rappelle Robert Marconis, « une agrégation féminine d'histoire et géographie, faisant place à l'histoire de l'art, n'en fut pas moins maintenue jusqu'en $1970 »(1)$.

Fruit d'un long processus qui a culminé entre 1941 et 1943, la création de l'agrégation de géographie est située par quelques-uns en 1940 (2), peut-être pour ne pas l'associer au régime de Vichy. P. Claval la place en 1944 (3) évoquant peut-être les sept premiers agrégés de géographie reçus au titre de l'année 1944, dans un concours reporté au début 1945. De Martonne présida les deux premières sessions. Revendication ancienne, l'agrégation de géographie a vu, après quelques péripéties, ses épreuves définies par l'arrêté de septembre 1943; elles resteront identiques jusqu'à la fin du $\mathrm{XX}^{\mathrm{e}}$ siècle. Les circonstances étaient favorables, compte tenu de l'estime des gouvernants pour la géographie et pour certains de ses maitres. L'élection d'E. de Martonne à l'Académie des sciences, le 27 octobre 1942, témoigne de ce vif intérêt pour la géographie: c'est le premier géographe français qui « ait eu l'insigne honneur d'entrer dans l'illustre assemblée. Par ce geste, l'Académie des Sciences a voulu souligner la haute valeur qu'elle attribue à l'œuvre et l'estime particulière dans laquelle elle tient la personnalité scientifique. C'est un hommage qui rejaillit sur la géographie française », écrit Cholley (4).

(1) Robert Marconis, Introduction à la géographie, Paris, Armand Colin, 1996, p. 20.

(2) Inspection générale de l'éducation nationale, Groupe histoire et géographie, " Des repères indispensables dans un monde en mouvement », Bulletin officiel de l'Éducation nationale, ${ }^{\circ} 15,14$ avril 1994, pp. 1086-1087.

(3) P. Claval, op. cit., p. 226.

(4) A. Cholley, « La remise à M. de Martonne de son épée d'académicien », Annales de géographie. Bulletin de la Société de géographie, $\mathrm{n}^{\circ} 292$, octobredécembre 1943, pp. 308-310. 


\section{VICHY : UN TEST ACIDE POUR LA GÉOGRAPHIE SCOLAIRE?}

Depuis des siècles, on publie des livres pour enseigner de la géographie, sa nomenclature, ses localisations, ses inventaires des richesses du monde et ses leçons sur les peuples. Cette éducation géographique contribue à la construction du rapport entre l'élève et son environnement, elle contribue à sa façon d'être au monde. Pour les éducateurs comme pour les politiques, il s'agit tout à la fois d'enseigner des faits et des valeurs. On comprend donc aisément que les gouvernants de Vichy aient fait preuve d'un vif intérêt pour l'enseignement de la géographie. Ils connaissaient la fonction identitaire de cette discipline scolaire. De plus, par contraste avec l'histoire, ses aspects qualifiés de concrets entraient en résonance avec l'idéologie pétainiste.

\section{Le rapport au monde et l'image des autres}

Ainsi, depuis longtemps, la géographie scolaire a construit des représentations de la France, des Français et des autres, images qui rétrospectivement nous semblent caricaturales, voire à connotations racistes. Au milieu du $\mathrm{XX}^{\mathrm{e}}$ siècle, le tempérament des peuples continue à être dépeint dans les livres de géographie. La collection Jean Brunhes, une des plus répandues, joint à son manuel pour la classe de troisième une sorte de codicille où les auteurs déclarent leur amour de la France.

« C'est le sens de la mesure qui est le fond du tempérament français. [...] Le Français n'a pas les préjugés de couleur qui caractérisent les Anglo-saxons; il admet les indigènes au bénéfice de sa culture, s'ils s'assimilent, il les considère comme de sa famille.

[...] La douceur de vivre, qui caractérise la France, la facilité cordiale de nos relations et notre respect pour autrui, nous ont conservé l'affection des Canadiens français et des Mauriciens, sujets de l'Angleterre et de nombreux habitants des Antilles. Notre influence est incontestable aussi sur la formation intellectuelle et morale des États latins de l'Amérique du Sud, dont les représentants vivent par milliers à Paris, qu'ils considèrent comme une seconde patrie. Les esprits libéraux subissent volontiers notre attirance et nous donnent leur sympathie. 
Descendants d'une race de paysans, nation encore à moitié paysanne, nous avons les qualités et les défauts des hommes dont les générations successives ont vécu du sol natal. Tout notre désir est de cultiver en paix notre jardin, la Douce France » (1).

Ce tableau moralisateur sera repris à l'identique dans l'édition de 1942, avec la même référence aux racines paysannes, avec aussi cette référence au libéralisme qui peut surprendre alors, et toujours cette tonalité implicitement antianglaise.

Cette animosité envers les Anglais n'est pas nouvelle et se retrouve dans les géographies scolaires du $\mathrm{XIX}^{\mathrm{e}}$ siècle. En ce qui concerne les années 1930-1940, l'étude de Marie-Claude BlancChaléard sur La vision du monde extérieur dans les manuels de géographie français avant et après la Seconde Guerre mondiale (2) montre cette persistance: une certaine anglophobie suinte des paragraphes qui sont consacrés « au caractère anglais ». Dans l'édition de 1937 du manuel de terminale de Gibert et Turlot, chez Delagrave, l'Anglais est décrit comme "pratique, égoïste et utilitaire, d'imagination lente [...], d'un orgueil prodigieux... » (3). Ces mêmes auteurs paraissent comme fascinés par l'Allemagne et les Allemands: "Toutes les forces ainsi organisées et réglées sont mises en mouvement vers un but précis, à la fois simple et pratique: depuis le chef de l'État jusqu'au dernier des citoyens, chacun se regarde comme l'ouvrier d'une œuvre commune, la grandeur de la Patrie allemande et la glorification de la Race germanique » (4). Ainsi, la géographie scolaire propose des jugements de valeur, proches des idées reçues; elle est discipline idéologique.

Les auteurs de manuels scolaires ne sont pas les seuls à diffuser une image des autres, négative ou positive, toujours comparative. Prenons comme exemple l'image des Juifs dans les volumes des

(1) Henri Boucau, Jean Petit, Armand Leyritz, Géographie, classe de $3^{e}$ et $3^{e}$ année des anciennes E.P.S. La France métropolitaine et la France d'Outre-mer. Nouvelle collection Jean Brunhes. Programmes des 11 avril 1938 et 26 octobre 1941, Paris, Hatier, 1941, pp. 325-326. La chanson Douce France de Charles Trenet et Léo Chauliac date de 1943.

(2) M.-C. Blanc Chaléard, op. cit.

(3) André Gibert, Gilbert Turlot, Principales puissances économiques du monde (moins la France). Cours de géographie M. Fallex, Paris, Delagrave, 1937, p. 24 (reproduit par M.-C. Blanc-Chaléard).

(4) Ibid., p. 177. 
années 1930 de la Géographie universelle. Augustin Bernard, membre de l'Institut, parle des " remarquables aptitudes commerciales » des juifs d'Afrique du Nord, développées par « un atavisme bien des fois séculaire » (1). E. de Martonne avait eu recours au même lexique pour présenter les Juifs en Europe centrale: ils commercent, sont redoutés et accaparent, écrit-il. Ils sont cantonnés dans des ghettos. Ils sont «étrangers », « allogènes », « inassimilables », « indésirables », « gênants »..., « ils affluent », répète-t-il. Il emploie à leur propos des mots lourdement chargés de sens comme « invasion », « infiltration », " problème ». Il présente la " question juive » en Europe centrale comme « un problème économique et non national » (2). Et quand de Martonne déplore le trop grand nombre de Juifs en Roumanie, il voit dans leur émigration une « solution » pour ce pays (3).

Ces mots résonnent tristement dans la France de Vichy. À partir du $\mathrm{n}^{\circ} 3$ de L'Information géographique, daté d'avril-mai-juin 1941, le nom de Jules Isaac est retiré de la liste des membres du comité de parrainage. Carcopino refuse d'intervenir pour soutenir Léon Abensour (4), qu'il avait reçu à l'agrégation d'histoire et de géographie en 1912 (5). En présentant la vie de Jean Gottmann, P. Claval écrit plutôt sobrement: "La guerre le contraint à chercher refuge aux États-Unis où il s'installe en 1941, se marie et se retrouve à l'université de Princeton » (6). Sans reprendre la biographie détaillée de ce grand géographe (7), rappelons qu'Albert Demangeon, gravement

(1) Augustin Bernard, « Afrique septentrionale et occidentale: Première partie: Généralités - Afrique du Nord », in Paul Vidal de La Blache, Lucien Gallois (dir.), Géographie universelle, t. XI, Paris, A. Colin, 1937, p. 88.

(2) Emmanuel de Martonne, «Europe Centrale, Suisse, Autriche, Hongrie, Tchécoslovaquie, Pologne, Roumanie » in P. Vidal de La Blache, L. Gallois (dir.), Géographie universelle, t. IV, vol. 2, 2 ${ }^{\mathrm{e}}$ partie, Paris, A. Colin, 1931, pp. 627-628.

(3) E. de Martonne, ibid., p. 708.

(4) Léon Abensour a publié en 1939 des manuels de géographie aux éditions Belin pour le primaire et le secondaire.

(5) Stéphanie Corcy-Debray, « Jérôme Carcopino et les lois d'exception », Revue d'histoire moderne et contemporaine, $\mathrm{n}^{\circ} 4,2002$, pp. 91-100.

(6) P. Claval, op. cit., p. 290.

(7) Emmanuelle Loyer, « La débâcle, les universitaires et la fondation Rockefeller: France/États-Unis, 1940-1941 », Revue d'histoire moderne et contemporaine, $\mathrm{n}^{\circ} 1$, 2001, pp. 138-159; Ron Johnston, Hugh Clout, Peter Hall, « Jean Gottmann, 19151994 », in Peter Armstrong, G. J. Martin (ed.), Geographers: Biobibliographical Studies, vol. 25, Londres, Continuum, 2006, pp. 42-59. 
malade, préoccupé par le sort de Gottmann, l'incite à quitter Paris pour se réfugier en zone non occupée, à Montpellier, auprès de son ami Jules Sion. Après la mort de Sion, Gottmann reste quelque temps à Montpellier, où il contribue au Bulletin de la Société languedocienne de géographie. En 1941, il réussit à passer en Espagne et ensuite à rejoindre les États-Unis où il se met, en tant que géographe, au service des Forces françaises libres. En 1944, il est nommé représentant provisoire de la République française aux Antilles, puis retourne en 1945 en métropole, où il conseille le ministre de l'économie Pierre Mendès-France et son successeur René Pleven.

Pendant ces dures années, on ne constate pas de comportement homogène d'une corporation de géographes. Certains se replient sur l'expertise technocratique, d'autres utilisent de façon opportuniste la Révolution nationale pour " libérer la géographie »; certains mobilisent la géographie au service de la « Révolution nationale », d'autres rejoignent la Résistance (1). Mais pendant les années d'occupation, il y a de nombreux repliements individuels, comme celui de Lucien Gachon, dont les romans paysans chantent alors l'enracinement dans la petite patrie, le retour à la terre, la dénonciation de la France des villes et de la modernité. Gachon opère alors une sorte de glissement de la géographie agraire au « retour à la terre » pétainiste (2).

Les années de guerre sont aussi celles où la Délégation générale de l'équipement national (DGEN), structure administrative créée par une loi de Vichy en février 1941, emploie de jeunes agrégés de géographie, chargés de mission (Jacques Weulersse, Louis Chevalier, Pierre George) (3). Les études de ces géographes visent à proposer des solutions pour la décentralisation ou la déconcentration des

(1) Pierre Gourou est vice-président du Comité de Libération à Bordeaux, et Jules Blache préfet de Meurthe-et-Moselle après la Libération de Nancy (J. Gottmann, op. cit.; A. Perpillou, op. cit.).

(2) «Indéniablement séduit par l'idéologie des premiers temps de la Révolution nationale », dit de lui Pierre Cornu, qui ajoute en note de bas de page: «Acceptant bon gré mal gré la fermeture des écoles normales, il se bat pour la création, en remplacement, d'écoles spécifiquement rurales, permettant de former des instituteurs imprégnés des valeurs terriennes, et non plus déracinés par les humanités gréco-latines. Malgré des démarches nombreuses et quelques appuis à Vichy, il n'obtient qu'un accueil mitigé et sans conséquence pratique ». P. Cornu, «Lucien Gachon: un itinéraire entre géographie rurale et littérature agreste », Ruralia, 12/13, 2003.

(3) Membre du PCF de 1935 à 1956. 
usines (1). En 1942, Jean-François Gravier publie Régions et nations et Yves-Marie Goblet La formation des régions. Introduction à la géographie économique de la France (2). La guerre, ça sert à faire de la géographie

\section{Méthodes actives et finalités autoritaires}

Nombreux sont ceux qui voient dans la géographie une discipline qui contribuera à reconstruire moralement la France. C'est dans ce contexte idéologique qu'est mise en place par le gouvernement de Vichy l'École nationale des cadres. Cette école, qui a pour mission « la formation des chefs », est installée à Uriage sous la direction de Paul-Henry Chombart de Lauwe. De 1940 à 1942, comme le montre Jean-Louis Tissier, les méthodes géographiques y sont utilisées pour former les nouvelles élites (3). L'étude du terrain, réputé concret, les questionnaires d'enquête (4), réputés pratiques, l'action, le travail en équipe sous la direction d'un chef ont des vertus formatrices qui permettent de se réapproprier la France et de forger les cadres du pays. Les travaux des géographes, en particulier ceux de Pierre Deffontaines (5), tiennent une grande place dans les références bibliographiques. P. Deffontaines, R. Blanchard se rendent à Uriage. Tissier estime qu'au cours des années 1941 et 1942, près de 2800 stagiaires y ont été formés, et ont en particulier pratiqué l'enquête de terrain (6). La formation par la pratique de la géographie est alors partie prenante de la « Révolution nationale ».

(1) Isabelle Couzon, « La figure de l'expert-géographe au miroir de la politique d'aménagement du territoire en France (1942-1950) », in G. Baudelle, M.-V. OzoufMarignier, M.-C. Robic (dir.), Géographes en pratiques..., op. cit., pp. 159-171.

(2) C. Gosme, J.-L. Tissier, op. cit.

(3) J.-L. Tissier, op. cit.

(4) Paul-Henry Chombart de Lauwe, Pour retrouver la France. Enquêtes sociales en équipe, collection « Le Chef et ses jeunes », 1941. Remanié et republié en 1947 sous le titre Pour comprendre la France.

(5) Pierre Deffontaines, Petit guide du voyageur actif, Comment connaître et comprendre un petit coin de pays?, Issoudun, édition du Laboureur, 1938, réédité en 1939, $91 \mathrm{p}$.

(6) Fin 1942, les cadres d'Uriage P.-H. Chombart de Lauwe et Hubert BeuveMéry rejoignent la Résistance. 
La conjoncture politique est donc porteuse pour la géographie, compte tenu du discrédit que les tenants de la «Révolution nationale » jettent sur l'histoire, mais cet engouement pour les études de terrain des géographes a des racines anciennes. L'enquête géographique est portée par les pratiques du scoutisme et des mouvements d'éducation nouvelle, et par les pédagogues partisans des méthodes actives. L'intérêt pour cette géographie de terrain date d'avantguerre. La création des activités dirigées par Jean Zay en 1938 est une incitation à mettre en place un enseignement « par l'aspect et par l'action ». L'enthousiasme pour la géographie se marque par de nouvelles publications: création en 1936 de la revue L'Information géographique (1), publication l'année suivante d'un numéro des Cahiers de pédagogie moderne pour l'enseignement du premier degré consacré à la géographie, avec les contributions de M. Sorre (2) et d'A. Demangeon (3), et d'un Vade-Mecum pour l'enseignement de la géographie de René Ozouf (4). C'est l'époque du Front populaire, des congés payés, du tourisme vert et des activités de nature.

L'intérêt pour la géographie locale, caractéristique de la géographie au temps de Vichy, est lui aussi en continuité avec l'avantguerre. La réhabilitation de l'histoire et de la géographie locales, des dialectes provinciaux, de la littérature de terroir (circulaire du 9 octobre 1940) peut être rapprochée du décret du 19 juin 1937, qui prévoyait la « création des ateliers-écoles près des établissements scolaires [...] qui devraient éveiller les aptitudes et la curiosité des élèves, ouvrir plus largement à la vie le travail scolaire, leur faire connaître l'histoire et la géographie locales ».

Il y a continuité avec la pratique des activités dirigées quand le Bulletin de l'Association de géographes français de décembre 1940 rapporte que « M. Emm. De Martonne se demande si la place de la géographie dans l'enseignement secondaire ne pourrait être élargie en

(1) Elle précède de deux années la création de l'Information historique.

(2) M. Sorre, «L'enseignement de la Géographie », in M. Sorre (dir.), Cahiers de pédagogie moderne pour l'enseignement du premier degré, Paris, Bourrelier, 1937, 114 p. (réédité en 1939).

(3) A. Demangeon, « Du rôle de la géographie dans l'enseignement », in M. Sorre (dir.), Cahiers de pédagogie..., op. cit.

(4) René Ozouf, Vade-Mecum pour l'enseignement de la géographie, Paris, Nathan, 1937, 128 p. 
la faisant intervenir dans les exercices physiques auxquels on veut donner une plus grande importance, une partie des horaires de ces exercices étant réservés à des excursions. Les camps de vacances pourraient aussi se prêter à la pratique de la géographie en plein air » (1). Et quand $\mathrm{P}$. George publie en 1942 À la découverte du pays de France. La nature et les travaux des hommes (2), Henri Boucau indique dans la préface que ce livre est écrit « pour le plein air, pour le promeneur solitaire, pour les routiers, pour les campeurs, pour les élèves de nos établissements que les séances d'enseignement général sortiront des habituelles murailles et verrières où s'enferme la majeure partie de leur existence scolaire ».

Certes, des différences de sensibilité apparaissent dans les principales collections de manuels scolaires des années 1930 et 1940 (3). La morale catholique est fort présente dans les livres pour l'école primaire de Jean Brunhes (4), moins nettement dans la collection qu'il dirige pour l'enseignement secondaire (5). La collection Demangeon, chez Hachette, est moins marquée politiquement (6). L'influence du marxisme et de l'école des Annales se manifeste dans les manuels de la collection Cholley.

A. Cholley (1886-1968) est un des fondateurs, en 1936, de la revue L'Information géographique, chez Baillière. Son titre complet, L'Information géographique pour l'enseignement, explicite son public et ses objectifs. Cholley en est le directeur avant, pendant et après la guerre. Il publie chez le même éditeur une collection de manuels scolaires pour le secondaire où écrivent des auteurs qui

(1) Bulletin de l'Association de géographes français, novembre-décembre 1940, p. 74.

(2) Pierre George, À la découverte du pays de France. La nature et les travaux des hommes, Paris, Bourrelier, 1942, $158 \mathrm{p}$.

(3) M.-C. Blanc-Chaléard, op. cit.

(4) À partir de 1925, chez Mame, à Tours.

(5) Brunhes meurt en 1930, son travail est poursuivi par sa fille Mariel JeanBrunhes Delamarre. Les manuels écrits sous son nom chez Hatier sont rédigés par André Allix (classe de terminale), Édouard Bruley (cinquième), Henri Boucau (seconde, première), etc. La «nouvelle collection » est publiée jusqu'en 1960.

(6) Les premiers livres sont publiés en 1938. Louis François dirige ceux de troisième et de première, A. Meynier celui de terminale. A. Demangeon décède en 1940. La collection est publiée jusqu'en 1961. 
furent membres du PCF, comme Pierre George et Jean Dresch (1). Marie-Claude Blanc-Chaléard caractérise leurs livres comme plus scientifiques par leurs contenus, plus arides dans leur mise en page et plus orientés vers l'analyse de l'espace en tant que production sociale (2). En 1941 Cholley rédige dans L'Information géographique un article à l'allure de manifeste. Dans un moment de probable désarroi idéologique, il glorifie une éducation géographique pour le moins directive (3).

\section{«LA GÉOGRAPHIE ET LA JEUNESSE}

La jeunesse dont nous examinerons le cas, au cours de cet article, n'est pas celle des Écoles, mais celle qui se trouve mise de bonne heure en contact avec les dures réalités de l'existence: la jeunesse des champs, celle des métiers et des usines.

Nous devons de plus en plus nous préoccuper d'elle; car, elle aussi, mérite d'être guidée, façonnée intellectuellement; nous avons plus que jamais besoin de toutes nos valeurs. [...]

Notre géographie n'en est plus à l'époque des synthèses hardies, des rapprochements audacieux et superficiels. Elle a depuis longtemps abordé la dure réalité des constructions patientes, ordonnées, exigeant des matériaux convenablement choisis et disposés selon un plan réfléchi, établi à la mesure du réel.

Bref, une dure école, d'où la facilité est exclue. [...]

Nous ne cesserons pas de protester contre le fait que l'enseignement de la géographie soit coulé dans le même moule que celui de l'histoire ou de la littérature. [...]

Ce qu'il faut éviter avant tout c'est, à ce sujet, de tomber dans l'étude des abstractions telles que l'industrie, l'agriculture, etc. Ce qui est la réalité même, ce sont les milieux divers créés par ces formes d'activité ou transformés par elle. Je préfère de beaucoup, à l'étude de l'agriculture en France, celle des divers milieux paysans adonnés à la culture; paysans ou fermiers de Beauce, de la Lorraine, du Midi aquitain; à celle de l'élevage, l'étude des divers milieux d'éleveurs: éleveurs de Normandie, du Limousin, de la Brie... [...]

(1) Y collaborent aussi Pierre Birot, René Clozier, Maurice Debesse, Marie-Laure Debesse-Arviset. Les premières éditions datent de 1935, les livres seront réédités jusqu'en 1954.

(2) M.-C. Blanc-Chaléard, op. cit., p. 38.

(3) En 1943, Cholley est le seul professeur d'université membre de la commission consultative chargée de proposer une liste des livres scolaires interdits dans l'enseignement primaire (arrêté du 19 juillet 1943). 
Observation, enquêtes, causeries solidement appuyées par une représentation cartographique, un bon choix d'images et de croquis; ce qui est bien là la meilleure documentation géographique. Nous croyons aussi que la réunion et la collaboration d'équipes diverses équipes d'étudiants, équipes paysannes, équipes ouvrières donneraient des résultats intéressants.

Les moniteurs. - La tâche de diriger une initiative ainsi conçue n'est évidemment pas des plus faciles. Elle ne peut être entreprise avec succès que si l'on dispose de véritables animateurs mais aussi de chefs ou de moniteurs fortement imprégnés de la méthode géographique. En lisant ces lignes on pourra peut-être penser que nous nous inspirons fortement du programme que l'on a appelé géographie locale. Dans une certaine mesure évidemment, puisque c'est l'observation et l'étude des faits locaux qui doit constituer le point de départ, la base même de notre construction géographique. Mais cette étude des faits locaux n'est pas aussi facile qu'on le croit communément. Le choix qui les retient est extrêmement délicat; il suppose non seulement une grande expérience de la région, mais il exige une culture très poussée en matière de géographie générale, culture à notre avis, impossible à acquérir en dehors d'un entraînement spécialisé. Autant dire que la formation des moniteurs ou des chefs d'équipe ne devrait être confiée qu'à des géographes jeunes et avertis » (1).

Ce discours programmatique en faveur de l'éducation géographique est plein d'ambiguïtés. Il entre alors en résonance avec l'idéologie maréchaliste, avec ses paysanneries locales, ses équipes composées de façon corporative par profession, sa hiérarchie de chefs et de moniteurs. Il définit clairement la géographie comme la découverte de la « réalité » (le mot revient quatre fois dans le texte), du « réel» (ce mot revient aussi quatre fois dans l'article), par opposition aux « synthèses hardies et aux rapprochements audacieux et superficiels ». Est-ce la géographie d'autrefois qui est visée ici ou, sans le dire, l'enseignement de l'histoire? La pratique de l'observation et les enquêtes sont mobilisées pour " guider », "façonner » la jeunesse. Les méthodes sont certes actives, mais encadrées, par le questionnaire d'enquête et sa grille d'observation et par les chefs ou moniteurs qui font les mises au point et guident les travaux. Ce n'est pas

(1) A. Cholley, « La géographie et la jeunesse », L'Information géographique, juin 1941, vol. 3, pp. 56-58. 
la créativité mais l'efficacité immédiate qui est primordiale. Les méthodes actives paraissent plus directives dans le contexte de 1941 qu'en 1937. Pédagogie active et idéologie autoritaire se combinent à Uriage, mais le goût de l'effort, du risque et de la vie de groupe était déjà promu au temps du Front populaire et des loisirs dirigés (1).

\section{La géographie scolaire et les héritages des années 1930 et 1940}

Si les programmes scolaires de Vichy donnent encore plus que les précédents à la France la place de pivot de l'ensemble du curriculum de géographie, il faut néanmoins souligner que ce n'est qu'une inflexion par rapport aux programmes précédents. La place importante qu'ils accordent à l'étude de l'empire colonial n'est pas en soi une nouveauté. Un article de Pierre et Marcel Clerget explicitement intitulé «Comment faire pénétrer par l'enseignement la notion d'empire français » le montre bien (2). Publié dans le premier numéro d'après-guerre de l'Information géographique, lorsque la revue reparaît, l'article semble avoir été rédigé pendant la guerre; il ne fait pas allusion à l'idée d'une France d'Outre-mer de 1945 et son titre sent la pédagogie directive, si ce n'est autoritaire. En fait, P. et M. Clerget ne font que présenter leur propre ouvrage, La France dans le monde, paru en 1938 (3). Cet exemple illustre la place centrale dans les programmes scolaires de l'étude de la France et de son empire, avant, pendant et après la guerre.

Même les formulations des manuels scolaires ne changent guère pendant l'époque de Vichy. Certes, les grands découpages en chapitres sont revus quand les programmes de 1943 répartissent autrement les questions précédemment étudiées. Mais les paragraphes sur la géographie physique sont conservés et, surtout, la géographie humaine des livres semble figée dans la situation d'avant-guerre. Les mêmes photographies de ports de commerce actifs illustrent les livres. L'appareillage statistique continue à reprendre les chiffres des années 1930, pour le commerce mondial comme pour les productions

(1) O. Dumoulin, op. cit., p. 31.

(2) Pierre Clerget, Marcel Clerget, « Comment faire pénétrer par l'enseignement la notion d'empire français ", L'Information géographique, avril 1942-juin 1945, n 3 4, pp. 60-62.

(3) Pierre et Marcel Clerget, La France dans le monde, Payot, 1938, 282 p. 
et les chiffres de population. Seules, les pages décrivant l'administration du pays connaissent quelques modifications, mais de façon relativement tardive. Ainsi, on peut lire dans l'édition de 1941 du livre de troisième de la collection Jean Brunhes (programmes de 11 avril 1938 et 26 octobre 1941, est-il indiqué en couverture): «Le gouvernement central. - Il réside à Paris et comprend des organes législatifs et exécutifs. Le pouvoir législatif (faire les lois) appartient au Parlement, divisé en Chambre des Députés et Sénat; la première est élue au suffrage universel, le second au suffrage restreint »(1). Une note de bas de page précise laconiquement: «Une modification constitutionnelle, non encore terminée, s'est produite en juillet 1940 ». Il faut attendre l'édition suivante, surtitrée « Programme du 23 décembre 1941 » et publiée en 1942, pour lire à cette même place: « Le gouvernement central. - Les désastres de Mai et Juin 1940 ont emporté la République démocratique, issue de la constitution de 1875. Elle est remplacée par un gouvernement autoritaire qui réside provisoirement en zone non occupée à Vichy » (2). La République ne semble pas avoir été retirée rapidement et avec entrain des livres de géographie.

Il faut de plus tenir compte de la faiblesse générale de l'édition scolaire en ces temps de guerre; les institutions et les particuliers sont terriblement appauvris et le papier manque. Marie-Claude BlancChaléard a fait un recensement, sans prétention à l'exhaustivité mais fort significatif, des vagues des éditions annuelles de manuels de géographie entre 1936 et 1948 (3). Elle montre que les publications nouvelles sont particulièrement nombreuses en 1939: il s'agit de la mise en œuvre des nouveaux programmes pour l'enseignement secondaire. La base de recensement des livres scolaires Emmanuelle confirme cette tendance. Alain Choppin y indique 69 éditions de titres de géographie en 1939, mais seulement 20 en 1940, 36 en 1941, 17 en 1942, 41 en 1943 et 15 en 1944. Ces chiffres incluent tous les ouvrages signalés en géographie scolaire, y compris les petites géographies départementales. Ils ne sont pas exhaustifs, ne prenant pas en compte les éditions non répertoriées et les volumes des différents tirages, mais ils constituent un échantillon très représentatif des tendances de l'édition scolaire de livres de géographie. Leur publication ne refleu-

(1) H. Boucau, J. Petit, A. Leyritz, op. cit., 1941, pp. 122, 126 et 127.

(2) Ibid, édition de 1942.

(3) Op. cit., annexes. 
rit qu'à partir de 1947, et, cette fois, tant pour le primaire que pour le secondaire. La base de données Emmanuelle signale 59 éditions de livres de géographie scolaire en 1947, et 89 en 1948.

Outre les problèmes matériels de l'édition en temps de guerre, le temps a heureusement manqué à Vichy, les nouveaux programmes n'étant applicables pour l'année scolaire 1943-1944 qu'en sixième, cinquième et première, et l'été 1944 ayant coupé court à la suite de cette entreprise. Ces programmes scolaires ont donc eu un impact limité, en particulier par leur courte durée. Rapidement, à la Libération, le programme de 1923 est repris pour le primaire, et le curriculum de 1938 est rétabli dans l'enseignement secondaire. Mais, changement d'importance, la géographie y fait désormais jeu égal avec l'histoire en termes d'horaire, du moins d'horaire prescrit. Ainsi la mobilisation idéologique de la géographie par la Révolution nationale vichyste n'a finalement pas desservi la discipline scolaire et universitaire qui se retrouve dotée après ces années de guerre d'une licence et d'une agrégation distinctes de celles de l'histoire.

Quelle que soit l'efficacité du travail de lobbying d'E. de Martonne auprès des gouvernants, le caractère durable de ce succès a été garanti par un contexte doublement porteur dans l'institution scolaire et dans le monde des universitaires. Du côté de l'institution scolaire, les partisans de la pédagogie par l'intuition et des pédagogies actives trouvaient dans la géographie une matière pour les leçons de choses, les classes-promenades, les enquêtes. L'intérêt pour les méthodes actives en géographie s'inscrit dans un mouvement de plus longue durée, fortement impulsé sous le Front populaire. Du côté de la géographie universitaire, pendant la guerre et dans l'après-guerre, nombre de géographes jouent un rôle important dans les organismes de conseil, de planification, de reconstruction. Si l'on ajoute à cela la renommée mondiale de l'école française de géographie, on comprend que ce qui était au départ l'instrumentation de la géographie au service de l'idéologie pétainiste se soit transformé en promotion de la géographie scolaire et de la géographie universitaire. Désormais la géographie scolaire fait, théoriquement, jeu égal avec l'histoire.

Jean-Pierre CHEVALIER Université de Cergy-Pontoise IUFM de l'académie de Versailles. E.H.GO., UMR 8504, Géographie-cités. 